Gwinner, H. von, Rauchstr. 1, Berlin (durch W. Scböller und R. Lepsius);

Ihlder, Dr. H., Wildau, Kr. Teltow (durch A. Bannow und F. Wilcke);

Bilecki, R., Boppstr. 4, Berlin

Häberle, R., Kurfürstenallee 38 , Charlottenburg

Wassuchnow, A., Roscherstr. 15, Charlottenburg

Weinberg, W., Achenbachstr. 9, Berlin W.

Frl. Traube, A., Bamberger Str. 53, Berlin W.

(durch

A. Rosenhein und

R. J. Meyer);

Rollett, Dr. A., Waldallee, Schwanhein b. Frankfurt a. M. (durch H. Steudel und R. Pschorr);

Reich, P., Quitzowstr. 108, Berlin NW. (durch S. Gabriel und R. Pschorr).

Für die Bibliothek sind als Geschenke eingegangen:

106. Jahresbericht über die Fortschritte der Chemie und verwandter Teile anderer Wissenschaften, begründet von J. Liebig und H. Kopp, herausgegeben von J. Tr̀ōger und E. Baur. Für 19051908. Heft 18 und 19. Braunschweig 1910.

Der Vorsitzende:

Der Schriltführer:

H. Wichelhaus.

F. Mylius.

\title{
Protokoll
}

äber die Sitzung des Ausschusses zur Wahrung der gemeinsamen Interessen des Chemikerstandes zu Berlin im ${ }_{n}$ Hôtel Continental ${ }^{*}$

am 14. Uktober 1910 , vormittags $11 \mathrm{Uhr}$.

Anwesend sind:

Geh. Oberregierungsrat Prof. Dr. K. v. Buchka) für die freie VereiniProf. Dr. A. Beythien

Prof. Dr. A. Bömer gung Deutscher Nabrungswittelchemilier;

Geb. Regierungsrat Prof. Dr. C. Duisberg für den Verein Deutscher Chemiker;

Holrat Dr. A. Forster für den Verband selbständiger öffentlicher Cbemiker;

Geb. Regierungsrat Prof. Dr. Marckwald für die Deutsche Chemische Gesellschaft. 
Den Vorsitz führt Professor Dr. Bömer, das Protokoll Professor Dr. Beythien.

Der Vcrsitzende eröffnet die Sitzung, begrüßt die Erschienenen und widmet sodann dem am 17. August d. J. verstorbenen Mitgliede des Ausschusses, Hrn. Dr. J. Treumann, der seit der Gründung des Ausschusses diesem als Mitglied angehört hat, warme Worte der Anerkennung für seine Tätigkeit im Ausschusse und gibt der Gewißheit Ausdruck, da $B$ die Mitglieder des Ausschusses ihm ein ehrendes Andenken bewahren werden. Er teilt ferner mit, daß an Stelle des Verstorbenen Hr. Professor Dr. Hintz-Wiesbaden vom Verbande selbständiger öffentlicher Chemiker in den Ausschuß gewählt sei.

I. Eingaben an das Reicbsamt des Innern und das PreaBische Kultusministerium. Der Vorsitzende verliest den Wortlaut der am 13. September d. J. abgegangenen Eingaben, die der Ausschu $B$ in seiner letzten Sitzung ${ }^{1}$ ) beschlossen batte. Um diese Fingaben durch mündliche Aussprache zu erläutern und zu ergänzen, begaben sich die Teilnehmer nach Schluß der Sitzung in die genannten Behörden, wo sie in sehr zurorkommender Weise empfangen wurden und Gelegenheit fanden, die Wünsche des durch den Ausschu $\beta$ vertretenen Chemikerstandes vorzutragen.

II. Seitens eines Mitgliedes des Ausschusses wird auf die Unzuträglichkeiten hingewiesen, die dadurch verursacht werden, $\mathrm{daB}$ auch immature Studierende der Chemie zum Verbandsexamen zugelassen werden. Diese Cbemiker bezeichneten sich, namentlich, wenn sie den Doktorgrad nicht erlangt hätten, später in den Fachzeitschriften bei Stellengesuchen und ähnlichen Anlässen vielfach als »Verbandschemiker" und suchten dadurch offenbar den Anschein zu erwecken, als ob sie ein staatliches Examen abgelegt hätten. Der AusschuB beschließt, an den Vorsitzenden des $\gg$ Verbandes der Laboratoriumsvorstände an deutschen Hochschulen« das Ersuchen zu richten, im Interesse der Hebung des Chemikerstandes nur mature Studierende der Chemie zum Verbandsexamen zuzulassen.

III. Veröffentlichung der Ausschuß-Protokolle. Der Ausschuß beschließt, bei dem bisherigen Gebrauche zu bleiben, die Protnkolle mōglichst am gleichen Termine nur in den Organen der im Ausschusse vereinigten Verbände zu veröflentlichen und der übrigen Fachpresse den Abdruck der Protokolle nach diesen Veröffentlichungen anheimzugeben.

Professor Dr. Bömer.

Dr. Beythien.

3) Vergl. diese Borichto 43, 2075-2077 [1910]. 
Die sub I erwäbnten Eingaben haben den folgenden Wortlaut:

Betrifft Ncuregelung der

Münster, den 13. September 1910.

Prüfungsordnung für

Nahrungsmittelchemiker.

Ew. Exzellenz

beehrt sich der unterzeichnete $»$ Ausschuß zur Wabrung del gemeinsamen Interessen des Chemikerstandes $\%$, in dem vertreten sind:

die Deutsche Chemische Gesellschaft*,

die Freie Vereinigung Dentscher Nahrungsmittelchemiker«,

der »Verband selbständiger öffentlicher Chemiker Deutschlands",

der Verein Deutscher Chemiker

und der daher eine Vertretung sāmtlicher deutschen Chemiker darstellt, das Nachstehende ehrerbietigst vorzutragen.

Während bei allen anderen gelehrten Berufen das Roifezeugnis einer neunklassigen höheren Schule ohne jede A tusnahme die Grundbedingung für die Zulassung zum Studium ist, soll nach zuverlässigen Nachrichten die Reichsregierung bei der in Schwebe begriffenen Neuregelung des Examens für Nahrungsmittelchemiker, des einzigen bis jetzt bestchenden Staatsexamens, für Chemiker, beabsichtigen, dio bisher bestehendea Ausnahmen för die $\mathrm{Zu}-$ lassung immaturer Apotheker beizubehalten.

Der ebrerbietigst unterzeichnete Ausschuß ist der Ansicht, daß mit Rücksicht auf die Schwierigkeit des Chemicstudiums und die große Bedeutung der Chemie in Wissenschait und Praxis die unbedingt notwendige gründliche Vorbildung durch das Reifezeugnis einer neunklassigen büheren Schule nachgewiesen, und $\mathrm{da} B$ daher dicses Reifezeugnis auch ohne $A$ usnahme als Grundbedingung für die Zulassung zum Examen für Nabrungsmittelchemiker gefordert werden müsse. El glaubt, daß diese Forderung um so mehr berechtigt ist, als auch die deutschen Universitäten und Hochschulen für die Verleihung eines akademischen Grades nunmehr allgemein die Maturităt als Grundbedingung fordern.

Aus den vorgetragenen Gründen bittet der Ausschuß Ew. Exzellenz, diesem auch bereits von verschiedenen anderen Chemikerkreisen an das Reichsamt cles Innern gerichteten Gesucho bei der in Aussicht stehenden Neuregelung des Examens für Nahrungsmittelchemiker geneigtest Folge geben zu wollen.

In Ehrerbietung

der $\gg A$ usschuß zur Wahrung der gemeinsamen Interessen des Chemikerstandes\&.

Im Auftrage

der z. Z. geschüftsfïhbrenden Freien Vereinigung Deutscher Nahrungsmittelchemiker gez. Prof. Dr. Bümer.

An

den Kaiserlichen Staatssekretär des Innern, Königlichen Staatsminister

Hrn. Delbrück, Exzellenz, Berlin. 
Betriflt Beanfsichtigung der

Münster, den 13. September 1910.

nichtstaatlichen óffentlichen

Untersuchungsanstalten.

Ew. Exzollenz

beehrt sich der unterzeichnete AusschuB zur Wahrung der gemeinsamen Interessen des Chemikerstandes", in dem vertreten sind:

die wDeutsche Chemische Gesellschait $\star$

die »Freie Vereinigung Deutscher Nahrungsmittelchemiker«,

der »Verband selbständiger öffentlicher Chemiker Deutschlands«,

der Verein Deutscher Chemiker»

und der daher eine Vertretung sāmtlicher deutschen Chemiker darstellt, das Nachstehende ehrerbietigst vorzutragen.

Durch Ew. Exzellenz Runderlaß yom 2. März d. J., betreffend die Nahrungsmittelkontrolle, soll die Beaufsichtigung der nichtstaatlichen öffentlichen Untersuchungsanstalten bezïglich ihrer allgemeinen Einrichtungen und ibrer Uutersuchungstätigkeit durch den Regierungs- und Medizinalrat oder den ārztlichen Hilfsarbeiter bei den Regierungen erfolgen und nur unter bosonderen Umstanden auf Grund eines Antrages an Ew. Exzellenz ein chemischer Sachverständiger hinzugezogen werden.

Der ehrerbietigst unterzeichnete Ausschuß ist der Ansicht, daß bei der Beaufsichtigung der Untersuchungsanstalten bezüglich ihrer allgemeinen Einrichtungen und ihrer Untersnchungstatigkeit entweder der Beaufsichtigende dieselbe Ausbildung wie der Beaufsichtigte haben, d. h. in diesem Falle approbierter Nahrungsmittelchemiker sein muB, oder daß wenigstens bei der Beaufsichtigung ein approbierter Nahrungsmittelchemiker stets hinzugezogen werden muß und nicht nur unter besonderen Umstānden hinzugezogen werden kann. Es sei gestattet, darauf hinzuweisen, daB anch bei jeder Apothekenrevision durch den zuständigen Regierungs- und Medizinalrat ein approbierter Apothcker hinzugezogen wird. Wenn diese Zuziehung eines Fachmannes bei der Apothekenrevision im staatlichen Aufsichtsinteresse von Ew. Exzellenz für erforderlich gehalten wird, so erscheint dies bei der Beaufsichtigung der Nahrungsmittel-Untersuchungsanstalten noch viel mehr geboten, da hier die Beurteilung der Einrichtungen und der Tätigkeit noch bedeutend mehr spezielle Kenntnisse und Elfahrungen erfordert als die Revision einer Apotheke.

Der AusschuB gibt sich der Hoffnung hin, daB Ew. Exzellenz vorstehendes Gesuch einer geneigten Erwăgung unterziehen werden.

\section{In Ehrorbietung}

der Ausschuß zur Wahrung der gemeinsamen Interessen des Chemikerstandes $\alpha$.

Im Auftrage

der z. Z. geschäftsführenden Freien Vereinigung Deutscher Nahrungsmittelchemiker

An gez. Prof. Dr. Bömer.

den Königl. Preußischen Staatsminister,

Minister der geistlichen, Unterrichts- und

Medizinal-Angelegenhoiten,

Hrn. von Trott zu Solz, Exzellenz;

Berlin. 